\title{
LA VULNERABILIDAD COMO CATEGORÍA EN CONSTRUCCIÓN EN LA JURISPRUDENCIA DEL TRIBUNAL EUROPEO DE DERECHOS HUMANOS: LIIMITES Y POTENCIALIDAD
}

\author{
MARIACATERINA LA BARBERA ${ }^{1}$ \\ Universidad Nebrija \\ mlabarbera@nebrija.es
}

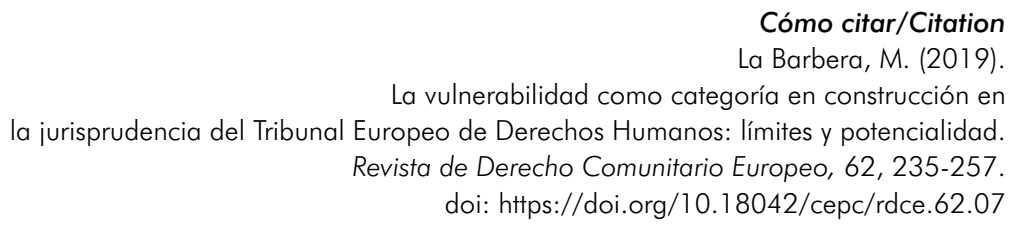

Resumen

Aunque el discurso de la vulnerabilidad es cada vez más frecuente en jurisprudencia, convenciones y recomendaciones internacionales, su significado está aún lejos de ser unívoco y coherente. Este trabajo analiza el concepto a de la vulnerabilidad en la jurisprudencia del Tribunal Europeo de Derechos Humanos (TEDH) de los últimos quince años. El objetivo es contribuir al debate académico sobre la vulnerabilidad como categoría que permite relacionar las violaciones de derechos humanos con sus causas estructurales en la praxis judicial, abriendo el camino hacía una protección sustantiva de los derechos humanos. Se destacan dos límites fundamentales del concepto de vulnerabilidad manejado por el TEDH: por un lado, la falta general de medidas positivas requeridas al Estado condenado y, por el otro, el uso de una noción naturalizada y homogeneizadora de grupo vulnerable. Finalmente, a través del

1 Profesora del Departamento de Derecho de la Universidad Nebrija, Madrid. Este trabajo se ha desarrollado en el marco de los proyectos DER2016-79805-P y RYC-2017-23010. Agradezco a Isabel Wences Simón y Juan Carlos Velasco Arroyo sus comentarios. 
análisis de la categoría de "vulnerabilidad específica», se identifica en el enfoque de la interseccionalidad un criterio interpretativo útil para explotar la potencialidad del concepto de vulnerabilidad en la praxis judicial del Consejo de Europa.

\section{Palabras clave}

Derechos humanos; discriminación; exclusión social; igualdad material; interseccionalidad; Tribunal Europeo de Derechos Humanos; vulnerabilidad.

\section{VULNERABILITY AS A CATEGORY UNDER CONSTRUCTION IN THE EUROPEAN COURT OF HUMAN RIGHTS JURISPRUDENCE: LIMITS AND POTENTIALITY}

Abstract

Although the vulnerability discourse is increasingly frequent in jurisprudence, international conventions and recommendations, its meaning is still far from being univocal and coherent. This work analyzes the concept of vulnerability in the jurisprudence of the European Court of Human Rights (ECtHR) in the last fifteen years. The goal of this work is to contribute to the academic debate on vulnerability as a category that allows human rights violations to be related with their structural causes in judicial practice, opening the door to a substantive protection of human rights. Two main limits of the concept of vulnerability that is handled by the ECtHR are addressed: on the one hand, the general lack of positive measures required to the condemned State and, on the other, the use of a naturalized and homogenizing notion of vulnerable group. Finally, through the analysis of the category of "specific vulnerability", the intersectionality approach is identified as a useful interpretative criterion to exploit the potentiality of the concept of vulnerability in the judicial praxis of the Council of Europe.

\section{Keywords}

Human Rights; discrimination; social exclusion; substantive equality; intersectionality; European Court of Human Rights; vulnerability.

\section{LA VULNÉRABILITÉ EN TANT QUE CATÉGORIE EN CONSTRUCTION DANS LA JURISPRUDENCE DE LA COUR EUROPEENNE DES DROTIS L'HOME: LIMITES ET POTENTIALITÉ}

\section{Résumé}

Bien que le discours sur la vulnérabilité soit de plus en plus fréquent dans la jurisprudence, les conventions et recommandations internationales, son sens est en- 
core loin d'être univoque et cohérent. Cet travail analyse le concept de vulnérabilité de la jurisprudence de la Cour européenne des droits de l'homme (CEDH) au cours des quinze dernières années. L'objectif de ce travail est de contribuer au débat universitaire sur la vulnérabilité en tant que catégorie permettant de relier les violations des droits de l'homme à leurs causes structurelles dans la pratique judiciaire, ouvrant ainsi la porte à une protection substantielle des droits de l'homme. Deux limites principales de la catégorie de vulnérabilité traitée par la Cour européenne des droits de l'homme sont abordées: d'une côté, l'absence générale de mesures positives imposées à l'État condamné et, de l'autre côté, l'utilisation d'une notion naturalisée et homogénéisante de groupe vulnérable. Enfin, à travers l'analyse de la catégorie de "vulnérabilité spécifique", l'approche de l'intersectionnalité est identifiée comme un critère d'interprétation utile pour exploiter le potentiel du concept de vulnérabilité dans la pratique judiciaire du Conseil de l'Europe.

\section{Mots clés}

Droits de l'homme; discrimination; exclusion sociale; égalité matérielle; intersectionnalité; Cour européenne des droits de l'homme; vulnérabilité. 


\section{SUMARIO}

I. INTRODUCCIÓN. II. LA EVOLUCIÓN DEL CONCEPTO DE GRUPOS Y PERSONAS VULNERABLES EN LA JURISPRUDENCIA RECIENTE DEL TRIBUNAL EUROPEO DE DERECHOS HUMANOS. III. LA VULNERABILIDAD ENTRE LAS CAUSAS ESTRUCTURALES DE LA DISCRIMINACIÓN Y LA CONTROVERTIDA DEFINICIÓN DE «GRUPO SOCIAL». IV. EL ENFOQUE DE LA INTERSECCIONALIDAD Y LA «VULNERABILIDAD ESPECÍFICA». V. CONCLUSIONES. BIBLIOGRAFÍA.

\section{INTRODUCCIÓN}

Basándose en los principios fundamentales de dignidad intrínseca e igualdad de todo ser humano, el derecho internacional de los derechos humanos tiene el objetivo de proteger los derechos de todas las personas y grupos, especialmente los más desaventajados. La concepción contemporánea de los derechos humanos se fundamenta en la consideración de los seres humanos como sujetos frágiles, interdependientes y necesitados de protección tanto frente a otros individuos como al Estado. Los derechos humanos son, ante todo, los derechos de la fragilidad humana y de su finitud (Viola, 2000; Grear, 2013). Como seres dotados de cuerpos y necesitados de relaciones sociales somos vulnerables.

En parte cada uno de nosotros se constituye políticamente en virtud de la vulnerabilidad social de nuestros cuerpos — como lugar de deseo y de vulnerabilidad física, como lugar público de afirmación y de exposición. La pérdida y la vulnerabilidad parecen ser la consecuencia de nuestros cuerpos socialmente constituidos, sujetos a otros, amenazados por la pérdida, expuestos a otros y susceptibles de violencia a causa de esta exposición (Butler, 2004: 46).

Por otro lado, cada persona experimenta la vulnerabilidad humana de forma única a través de cuerpos individuales y características particulares en relación con un conjunto complejo de causas que incluyen la situación económica, los estereotipos culturales y las condiciones físicas que pueden mermar nuestra capacidad de respuesta, adaptación y reacción frente a perturbaciones y situaciones naturales, sociales o institucionales adversas (Fineman, 2008, 2010 y 2013). Utilizar la vulnerabilidad en el contexto de las violaciones de 
derechos humanos pone de manifiesto la necesidad de reconceptualizar el sujeto político como un sujeto encarnado y situado en contextos geopolíticos, sociales e históricos concretos dentro de un complejo entramado de relaciones de interdependencia con los demás y con las instituciones (Gilligan, 1982; Pateman, 1983; Tronto, 1993; Nussbaum, 1999). Reconocer la vulnerabilidad es pues el paso necesario para la transformación social, pero depende esencialmente de las «normas de reconocimiento existentes» (Butler, 2004: 70-71).

Si el posicionamiento social depende de las instituciones y estructuras políticas y estas a su vez definen el acceso a los recursos, las opciones y oportunidades (Fineman, 2008), el contrapunto a la vulnerabilidad no es la invulnerabilidad sino la resiliencia, es decir, la capacidad de reaccionar y hacer frente a las adversidades, incluyendo las vulneraciones de derechos. Siendo la vulnerabilidad en gran medida determinada por la falta de recursos que permitan reaccionar ante la exposición al daño, el reconocimiento de la vulnerabilidad implica reconocer la necesidad de establecer obligaciones positivas por parte de las instituciones públicas que deben garantizar el acceso a dichos recursos (Fineman, 2010).

Aunque desde la adopción de la Declaración Universal de los Derechos Humanos en 1948 la vulnerabilidad es un concepto implícito en el discurso de los derechos humanos, desde principio de los años noventa se ha hecho cada vez más frecuente la referencia explícita a la noción de «vulnerabilidad» en convenios y recomendaciones internacionales. Así, la "Convención internacional sobre la protección de los derechos de todos los trabajadores migrantes y de sus familiares» (1990) se refiere a la situación de vulnerabilidad en la que con frecuencia se encuentran los trabajadores migrantes y sus familiares debido, entre otros, a su lejanía del país de origen y a las dificultades que encuentran en el país donde trabajan ${ }^{2}$. El «Protocolo facultativo de la Convención sobre los derechos del niño relativo a la participación de niños en los conflictos armados» (1995) reconoce las necesidades especiales de los niños, cuya situación económica, social y género les expone de manera especial a la vulneración de derechos a causa del reclutamiento o utilización en situación de hostilidad ${ }^{3}$. Asimismo, de acuerdo con el «Protocolo facultativo sobre la venta de niños, la prostitución infantil y la utilización de niños en la pornografía» (2000), los Estados partes deberán promover el fortalecimiento de la cooperación internacional para luchar contra aquellos factores, como la pobreza y el

2 Véase «Convención internacional sobre la protección de los derechos de todos los trabajadores migrantes y de sus familiares» en: https://bit.ly/2VkLq7h.

3 Véase Protocolo facultativo de la Convención sobre los Derechos del Niño relativo a la participación de niños en los conflictos armados en: https://bit.ly/2BTy4HI. 
subdesarrollo, que contribuyen en exponer a los niños a la venta, prostitución, pornografía y turismo sexual ${ }^{4}$. Finalmente, la «Convención internacional para la protección de todas las personas contra las desapariciones forzadas» (2006) establece que los Estados partes podrán establecer circunstancias agravantes en los casos de deceso de las personas desaparecidas o desaparición forzada de mujeres embarazadas, menores, personas con discapacidad u otras personas particularmente vulnerables 5 .

En sentido análogo, resoluciones no vinculantes del Parlamento Europeo reconocen la necesidad de otorgar especial protección a personas y grupos en situación de particular vulnerabilidad. Piénsese, por ejemplo, en la resolución de 2014 sobre violencia contra las mujeres (2013/2004/INL), que reconoce las especiales necesidades y la mayor vulnerabilidad de estas debido a factores como raza, etnia, religión o creencias, salud, estado civil, vivienda, estatus migratorio, edad, discapacidad, orientación sexual e identidad de género; o en la resolución sobre explotación sexual y prostitución (2013/2013/INI) que invita a los Estados miembros a adoptar políticas que ayuden a mujeres vulnerables y menores a salir de la prostitución a través de un enfoque integral que involucre la cooperación entre los servicios de policía, migración, salud y educación.

A pesar de su cada vez más explícito uso, el contenido de la vulnerabilidad como categoría jurídica es aún ambiguo. Si bien es posible que el éxito de esta noción radique precisamente en su vaguedad e indeterminación, la utilización en decisiones judiciales de categorías ambiguas conduce a menudo a fallos contradictorios y efectos perversos en su implementación. Estudios recientes han analizado el uso de la categoría de la vulnerabilidad en los documentos del Comité de Derechos Económicos, Sociales y Culturales (Chapman y Carbonetti, 2011), la Corte Interamericana de Derechos Humanos (Estupiñan Silva, 2014), el Tribunal Europeo de Derechos Humanos —en adelante TEDH o Tribunal- (Timmer, 2013; Peroni y Timmer, 2013) y en la legislación nacional, especialmente en Reino Unido (Munro y Scoular, 2012; Fitzgerald 2010). El tema ha pasado recientemente a ser objeto de reflexión de la teoría del derecho en España (Barranco y Churruca, 2014; Barrère Unzueta, 2016; Morondo Taramundi, 2016; Barranco Avilés, 2016). Este artículo pretende contribuir a este debate académico, adoptando la

4 Véase Protocolo facultativo sobre la venta de niños, la prostitución infantil y la utilización de niños en la pornografía en https://bit.ly/2CKtT0C.

5 Véase Convención internacional para la protección de todas las personas contra las desapariciones forzadas en https://bit.ly/2NyJEfr. 
perspectiva de género y analizando cómo este se entrecruza con otras situaciones de vulnerabilidad, en particular el estatus migratorio.

Este trabajo analiza la evolución no lineal de la vulnerabilidad como categoría en la jurisprudencia del TEDH y pretende contribuir a su desarrollo conceptual, destacando sus límites y potencialidad. El objetivo es afinar su capacidad operativa como categoría para la praxis judicial. Para ello, el artículo se estructura en dos partes. En la primera se ofrece una aproximación a la evolución del concepto de grupos e individuos vulnerables a través de casos judiciales recientes del TEDH que han utilizado la categoría de la vulnerabilidad para reconocer una violación del Convenio Europeo de Derechos $\mathrm{Hu}-$ manos (en adelante Convenio). Se discute, en particular, la relación entre las causas sistémicas de discriminación y la noción de grupo. En la segunda parte se analiza el concepto de "vulnerabilidad específica» desde el enfoque de la interseccionalidad. Se propone finalmente la interseccionalidad como criterio útil para explotar la potencialidad de la categoría de la vulnerabilidad en la protección sustantiva de los derechos humanos en la praxis judicial.

\section{LA EVOLUCIÓN DEL CONCEPTO DE GRUPOS Y PERSONAS VULNERABLES EN LA JURISPRUDENCIA RECIENTE DEL TEDH}

El TEDH aludió a la vulnerabilidad por primera vez en un voto disidente de Buckley c. Reino Unido (1996). En el caso Buckley, el juez eslovaco Bohumil Repik argumentaba, en su voto parcialmente disidente, que reconocer la vulnerabilidad de los gitanos como minoría implicaba tener en especial consideración sus necesidades y estilo de vida diferente tanto a la hora de definir el marco legislativo aplicable como en la decisión del Tribunal ${ }^{6}$.

En el mismo sentido, en Chapman c. Reino Unido (2001), los jueces disidentes, en su voto conjunto, argumentaron la existencia de una obligación positiva de los Estados contrayentes a facilitar el modo de vida gitano (Chapman c. Reino Unido, párr. 96). Los jueces disidentes añadieron que el Estado no solo tiene la obligación de evitar la discriminación formal en su ordenamiento,

6 «The Court, in order to fulfil its supervisory role, ought itself to have considered whether the interference was proportionate to the right in issue and to its importance to the applicant, all the more so as where a fundamental right of a member of a minority is concerned, especially a minority as vulnerable as the Gypsies, the Court has an obligation to subject any such interference to particularly close scrutiny. In my opinion, the Court has not fully performed its duty as it has not taken» (Buckley c. Reino Unido, voto parcialmente disidente del juez Repik). 
sino también comprometerse a eliminar las formas de discriminación materiales que obstaculizan de hecho el igual disfrute de derechos y libertades. El juez español Antonio Pastor Ridruejo, el maltés Giovanni Bonello, la belga Françoise Tulkens, la eslovaca Strážnická, el danés Peer Lorenzen, el luxemburgués Marc Fischbach y el andorrano Josep Casadevall se refirieron al consenso emergente entre los Estados miembros del Consejo de Europa sobre el reconocimiento de las necesidades especiales de las minorías y la obligación de proteger su seguridad, identidad y estilo de vida, no solo con el propósito de proteger los intereses de las minorías, sino también con el fin de preservar la diversidad cultural como valor de toda la comunidad. Estos argumentos allanaron el camino para casos siguientes en los que el Tribunal, considerando la posición vulnerable de la parte demandante, reconoció la existencia de violaciones de derechos protegidos por el Convenio.

A partir de esta primera elaboración, el TEDH ha empezado a relacionar de manera más frecuente las características asumidas como definitorias de un grupo en situación de vulnerabilidad con la violación de derechos sufrida. Asimismo, se han definido como vulnerables a presos [Salman c. Turquia (2000), Algür c. Turquía (2002)], menores no acompañados [Mubilanzila Mayeka and Kaniki Mitunga c. Belgium (2006)], personas con discapacidad intelectual [Shtukaturov c. Rusia (2007), Alajos Kiss c. Hungria (2010)], gitanos [D. H. y otros c. República Checa (2007), Orsus y otros c. Croacia (2010) y Horváth y Kiss c. Hungria (2013)], solicitantes asilo [M.S.S. c. Bélgica y Grecia (2011)], personas enfermas de VIH [Kiyutin c. Rusia (2011)], mujeres víctimas de violencia de género [Opuz c. Turkey (2009), Hajduova c. Eslovaquia (2010)], mujeres embarazadas [Svetlana Orlova c. Russia (2009), Nechiporuk y Yonkalo c. Ukraine (2011), P. y S. c. Poland (2012)] y con problemas de salud reproductiva [R.R. c Polonia (2011)]. Finalmente, más recientemente el TEDH ha utilizado el concepto de "vulnerabilidad específica» [B.S. c. España (2012)] aludiendo a una noción más compleja de vulnerabilidad vinculada con las privaciones materiales y contextuales entrelazadas (intersectional discrimination) que ha sufrido la demandante:

A la luz de los elementos de prueba proporcionados en este caso, el Tribunal estima que las decisiones dictadas por los Órganos Jurisdiccionales internos, no tuvieron en cuenta la vulnerabilidad específica de la demandante, inherente a su condición de mujer africana ejerciendo la prostitución [cursiva propia]. Las Autoridades faltaron así a la obligación que les incumbía, en virtud del artículo 14 del Convenio combinado con el artículo 3, de adoptar todas las medidas posibles para ver si una actitud discriminatoria hubiera podido, o no, desempeñar algún papel en los sucesos (B.S. c. España, párr. 71). 
De acuerdo con Lourdes Peroni y Alexandra Timmer (2013), el uso de la categoría de la vulnerabilidad permite al Tribunal empezar a abordar los derechos humanos, y en particular la igualdad y no discriminación (art. 14 $\mathrm{CEDH}$ ), en su dimensión material y transformativa. Por esta razón, a pesar de las cuestiones problemáticas que suscita, hay que reconocer en el uso de la categoría de la vulnerabilidad un avance positivo de la jurisprudencia reciente del TEDH. La novedad que aporta la utilización de la categoría de la vulnerabilidad es la identificación — aunque en ocasiones vaga - de acciones o inacciones que causan la violación de derechos o incrementan la exposición al riesgo de sufrirlo. En D. H. y otros c. República Checa, por ejemplo, la Gran Sala señala que, "como resultado de su historia turbulenta y desarraigo constante, los gitanos se han convertido en un tipo específico de minoría desfavorecida y vulnerable, y que debe darse consideración especial a sus necesidades y su estilo de vida diferente, tanto en el marco regulatorio aplicable como en la toma de decisiones en casos particulares» (párr. 181), debiéndose proporcionar "protección especial, en particular en el campo de la educación» (párr. $182)^{7}$. Utilizando el marco interpretativo de la vulnerabilidad, el TEDH diagnostica los problemas de violaciones de derechos fundamentales que sufren los niños gitanos y los vincula a su estilo de vida diferente y su condición de grupo minoritario marginado a nivel social e institucional. Sin embargo, en los fallos, solo en contadas ocasiones - por ejemplo, en Shtukaturov c. Rusia (2007) — se indica una solución o pronóstico — "what should be done?», para decirlo con Carol Bacchi (1999)—. Es decir, en la mayoría de las decisiones el TEDH no identifica los casos como casos excepcionales en los que puede indicar el tipo de medida que se debe adoptar para erradicar el problema estructural que produjo la violación de derechos reconocida y ayudar al Estado demandado a cumplir con sus obligaciones de ejecución de las sentencias (art. 46 ECHR). El Tribunal recuerda que sus sentencias tienen un carácter esencialmente declarativo y que en general le corresponde en primer lugar al Estado condenado, bajo el control del Comité de Ministros, elegir los medios que deben utilizarse, dentro del ordenamiento jurídico nacional, en observancia del art. 46 del Convenio [Scozzari y Giunta c. Italia (GC, 2000), párr. 249; Assanidzéc. Georgia (GC, 2004), párr. 202; B.S. c. España, párr. 80).

\footnotetext{
Por otro lado, en su opinión disidente, el juez español Javier Borrego Borrego ha puesto de relieve los límites del concepto de vulnerabilidad, criticando la posición de la mayoría que duda de la capacidad de ejercer la potestad parental por parte de los padres de etnia gitana, que él define como "fighting racism through racism» $(D . H . y$ otros c República Checa).
} 
La inconsistencia entre diagnóstico y pronóstico pone en evidencia un límite fundamental del uso de la categoría de la vulnerabilidad por parte de una institución internacional de derechos humanos como es el TEDH. El reconocimiento de una violación de los derechos reconocidos en el Convenio permite la compensación de daños materiales y no materiales y garantiza la tutela efectiva de dichos derechos. Sin embargo, el reconocimiento de que dichas violaciones se originan en factores histórico-sociales, y que estos son reproducidos por las instituciones políticas y el marco jurídico, implicaría como condición necesaria la identificación de una obligación positiva de eliminar los obstáculos que impiden alcanzar el disfrute de los derechos en igualdad de condiciones. Utilizar la vulnerabilidad como marco interpretativo de las violaciones de derechos humanos conlleva no solo incluir en la ponderación judicial las causas que generan la violación de los derechos humanos, identificando qué tipo de decisiones, acciones o inacciones se han tomado y por quién. Implicaría también definir cuál es la relación entre estas acciones u omisiones y las situaciones que generan el riesgo específico de vulneración de derechos para algunas personas o grupos de personas. Utilizar la categoría de la vulnerabilidad en las decisiones judiciales de un tribunal internacional requiere no limitarse a sancionar los Estados dónde se han cometido las violaciones identificadas, sino exigir que los Estados desempeñen un papel proactivo y adopten medidas positivas para promover los derechos humanos y erradicar las violaciones de derechos (Peroni y Timmer 2013: 1076). En otras palabras, adoptar la categoría de la vulnerabilidad impone consecuencias trascendentales para el derecho internacional de los derechos humanos, ya que requiere un papel propositivo y no solo reactivo de los jueces internacionales. En este sentido, la categoría de la vulnerabilidad requiere una transformación — compleja y lenta - sin la cual, sin embargo, la vulnerabilidad no puede desempeñar su potencial transformador y seguirá reproduciendo la otredad y la marginalización que pretende eliminar (Timmer, 2013, Peroni y Timmer, 2013). Hay que recordar que solo en algunas ocasiones excepcionales [Shtukaturov c. Rusia (2007), Alajos Kiss c. Hungría (2010)] el TEDH ha sido tajante a la hora de afirmar que el margen de apreciación del Estado es más reducido cuando se aplica una restricción de los derechos fundamentales a un grupo particularmente vulnerable que ha sufrido una considerable discriminación en el pasado, como las personas con (dis)capacidad mental. La doctrina de la vulnerabilidad del TEDH requeriría pues intervenir más a menudo en cuestiones consideradas de competencia nacional, poniendo al descubierto cuestiones relacionadas con la cesión de la soberanía nacional que no están resueltas ni en el seno del Consejo de Europa ni de la Unión Europea y que eventos recientes, como el brexit, han puesto nuevamente en el centro del debate público (Gyorfi, en prensa). 


\section{LA VULNERABILIDAD ENTRE LAS CAUSAS ESTRUCTURALES DE LA DISCRIMINACIÓN Y LA CONTROVERTIDA DEFINICIÓN $D E$ «GRUPO SOCIAL»}

La jurisprudencia reciente del TEDH vincula la vulnerabilidad con el contexto social, cultural, histórico e institucional que determina estas situaciones. Para entender la categoría de vulnerabilidad como sobreexposición al riesgo de sufrir vulneraciones de derechos humanos utilizada por el Tribunal es necesario pues desentrañar la relación compleja entre estructuras sociales y las situaciones individuales específicas que las generan (Peroni y Timmer, 2013: 1063). Ya que el TEDH no define claramente la línea de demarcación entre grupos e individuos vulnerables (Al Tamimi, 2016), abordar la categoría de la vulnerabilidad se encuentra en un nudo fundamental para la protección de los derechos humanos: la relación entre individualidad y colectividad ${ }^{8}$. El paradigma liberal de los derechos humanos confiere la titularidad de los derechos a los individuos. Sin embargo, las personas que se encuentran en situación de desventaja sufren violaciones de derechos a causa de un conjunto de factores sociales e institucionales que definen su condición social en base a estructuras colectivas — género, raza, posición económica, (dis)capacidad, orientación sexual, edad, origen nacional, religión- que definen el margen de acción y reacción individual.

Cuando ciertas condiciones personales son identificadas como factores que aumentan la exposición a la vulneración de derechos, y estas condiciones son comunes a varias personas, quienes han sufrido una vulneración son representados como integrantes de un grupo. El TEDH identifica como causas de la vulnerabilidad de determinados grupos la historia de estigmatización, segregación y exclusión, apuntando de esta forma a las estructuras sociales e institucionales que mantienen y reproducen su vulnerabilidad (D. H. y otros c. República Checa, Alajos Kiss c. Hungría). La categoría de "grupo vulnerable» implica la existencia de características que son compartidas entre los sujetos

8 La relación entre individualidad y colectividad se articula en el cruce entre la universalidad de los derechos humanos y la particularidad de los sujetos de derecho, de los contextos en los que se desarrollan sus vidas, de las condiciones específicas que les hacen vulnerables y necesitados de protección por parte de instituciones nacionales y supranacionales. Hablar de especial vulnerabilidad no significa hablar de derechos especiales de grupos y personas vulnerables, sino de establecer medidas especiales para reparar daños u otorgar satisfacción equitativa (art. $41 \mathrm{CEDH})$ a los que han sufrido vulneración de derechos humanos a causa de obstáculos estructurales específicos que les han impedido disfrutar, en igualdad de condiciones, de los derechos y libertades previstos como fundamentales para todos los seres humanos. 
que se representan como pertenecientes al grupo y que las diferencian del resto de la población y los ubican en una situación de mayor desventaja (Barranco Avilés, 2014). La categoría de la vulnerabilidad permite poner el foco en aquellas situaciones de marginalización, exclusión de la participación económica y política, empobrecimiento y falta de protección que generan la vulneración efectiva de quienes son potencialmente vulnerables (Anderson, 1994; Barrère Unzueta, 2016). La vulnerabilidad de un grupo es un indicador que revela una situación de riesgo, de sobreexposición al daño y mayor probabilidad de sufrir una vulneración de derechos. Sin embargo, es necesario considerar las situaciones contextuales y específicas que dan lugar a la vulneración efectiva de derechos individuales y colectivos. Raza, género, religión, sexualidad, edad y (dis) capacidad $^{9}$ han sido identificados como indicadores de vulnerabilidad (Chapman y Carbonetti, 2011). Sin embargo, al asumir estas características como «dadas» $\mathrm{y}$ «naturales», se ignoran los procesos de construcción de estas estructuras sociales que definen la posición individual. Es decir, se hace referencia a las estructuras de organización social en términos dicotómicos y jerárquicos (blanco/negro, hombre/mujer, cristianos/otros, heterosexuales, niños/adultos, personas física y mentalmente sanas/personas con discapacidad) como factores explicativos de la discriminación sistémica, pero no se indaga de qué manera se construyen, por ejemplo, el género (Scott, 1988; Young, 1990; Butler, 1990) o la raza (Omi y Winant, 1986; Perry, 2011).

La definición de un grupo depende de cómo se conteste a la pregunta "¿quiénes constituyen el grupo normativo de referencia?». La respuesta es inevitablemente relacional y contextual (La Barbera, 2015): se define como grupo a las personas que supuestamente no tienen las características definitorias de lo «normal», de la así llamada "población en general» (Philips, 2010; Barranco Avilés, 2014). De esta forma, la vulnerabilidad se define como variación, o mejor déficit, respecto a un paradigma considerado como referencia (Luna, 2009; Fineman, 2010; Timmer, 2013). El TEDH asume como estándar un sujeto adulto, autónomo, sin dependencias, con un adecuado nivel de instrucción, capacidades cognitivas inalteradas, libertad de movimiento y autodeterminación. A partir de esta definición apriorística del sujeto político

9 Aunque en la literatura más reciente se haga referencia al concepto de diversidad funcional (Rodríguez Díaz y Ferreira, 2010), he optado por utilizar el término «(dis) capacidad» no solo porque «discapacidad» es el término utilizado en la legislación, jurisprudencia y convenciones internacionales, sino también porque usar el término «(dis)capacidad» permite poner en evidencia la dimensión relacional del estigma y exclusión de la así llamada «discapacidad» con la condición de privilegio y poder invisibilizado que se esconde detrás de la «capacidad». 
titular de derechos como sujeto masculino, heterosexual, física y mentalmente hábil, blanco y nacional, se definen como grupos a las mujeres porque no son hombres, a las personas con (dis)capacidad por que no poseen las capacidades físicas consideradas «normales», a los negros porque no son blancos, a los migrantes porque no son nacionales. Por el contrario, los hombres nunca son grupo. Los blancos tampoco, así como no lo son los física y mentalmente sanos. Ellos son simplemente la norma.

Por otro lado, la definición de un grupo implica la asunción de cierta homogeneidad interna (Fineman, 2013) e ignora la existencia de diferencias significativas entre los que se representan como sus integrantes. En particular, ignora las diferencias de género cuando el grupo se define en base a la raza (Okin, 1999) o de raza cuando el grupo se define en base al género (Crenshaw, 1989). Se incurre en el mismo riesgo de homogeneización también cuando se usa la posición económica para definir un grupo como vulnerable, pues la categoría de la pobreza ignora las diferencias marcadas por el género y la etnia, a la vez que la categoría de estatus migratorio oculta las diferencias de posición económica, género, etnia, religión, origen nacional y color de piel.

En la jurisprudencia del TEDH se observa una tendencia a aplicar la vulnerabilidad como una "etiqueta» inmutable y rígida que ignora que una persona puede sufrir diferentes tipos de vulnerabilidades entrelazadas (Luna, 2009: 326). Sin embargo, la esencialización de la vulnerabilidad naturaliza los procesos de construcción social a través de los cuales se define un grupo (Phillips, 2010: 58) y termina por reproducir estereotipos (Truscan, 2013). Estas aproximaciones homogeneizadoras y naturalizadas ignoran que dentro de un grupo existen «diferencias que marcan la diferencia» en la manera de vivir las experiencias de discriminación (Crenshaw, 2000) y generan resultados victimizadores y paternalistas que perpetuán las situaciones de discriminación de forma sutil y más compleja de desmantelar.

El TEDH, en este sentido, se encuentra ante una encrucijada. Al utilizar una noción naturalizada y homogeneizadora de grupo y al prever una satisfacción equitativa sin indicar, en la mayoría de los casos, los instrumentos que los Estados deben adoptar para evitar futuras violaciones, el Tribunal termina por utilizar «vulnerabilidad» como sinónimo de «debilidad» intrínseca, sin posibilidad de «salida». Erosionando la capacidad de acción institucional y agencia individual, la vulnerabilidad se convierte en estigma (Keogh et al., 2010; Peroni y Timmer, 2013; Da Lomba, 2014). Sin embargo, el concepto de la vulnerabilidad nace con la finalidad opuesta de eliminar el estigma y la discriminación para garantizar el disfrute sustantivo de derechos y libertades en igualdad de condiciones. Pero ello no es posible si no se pone el foco en los cambios sociales necesarios a la vez que se identifican los individuos que se deben proteger (Solanke, 2016: 6). 
En el caso de las personas con (dis)capacidad mental, el TEDH considera que estas han sido históricamente objeto de prejuicios - mantenidos y reproducidos por disposiciones legislativas que prohíben la evaluación individualizada de sus capacidades y necesidades- que determinan su exclusión social y tienen consecuencias duraderas (Alajos Kiss c. Hungría, párr. 42). En los casos Shtukaturov y Alajos Kiss los demandantes habían perdido su derecho al voto como resultado de la imposición de una restricción automática de su capacidad jurídica sin evaluación previa. El Tribunal consideró en ambos casos que el tratamiento de las personas con (dis)capacidad mental como grupo homogéneo se basa en una clasificación cuestionable, problematizando el uso de una noción homogeneizadora de grupo.

En su opinión parcialmente disidente en M.S.S. c. Bélgica y Grecia, el juez húngaro András Sajó argumenta que la construcción de los solicitantes de asilo como grupo vulnerable ignora las diferencias de estatus dentro del presunto "grupo" de los refugiados. En su opinión, los solicitantes de asilo difieren de los gitanos y de las personas con (dis)capacidad mental ya que no son un grupo históricamente sujeto a prejuicios que han dado lugar a su exclusión social de manera duradera. De acuerdo con el juez Sajó, el TEDH utiliza la categoría de grupo vulnerable cuando todos los miembros de un grupo, debido a su categorización social adversa, merecen una protección especial por parte del Estado que dispone, en estos casos, de un margen de apreciación sustancialmente reducido. Según el juez Sajó, los solicitantes de asilo, como grupo, no estarían incapacitados ni habrían perdido el control sobre su propio destino.

Por otro lado, el juez Sajó considera que el efecto psicológico negativo causado por las disfunciones del sistema de asilo define la posición de vulnerabilidad de los solicitantes de asilo. En línea con la jurisprudencia del TEDH sobre vulnerabilidad de los presos [Davydov y otros c. Ucrania (2010, párr. 202), la dependencia total para la validación de su capacidad de participar en la sociedad de acogida puede definirse como vulnerabilidad debido a las limitaciones impuestas por el Estado. Incluso el solicitante de asilo con más recursos no puede otorgarse a sí mismo la condición de refugiado ni conseguir acceso a los derechos en la sociedad de acogida (Clayton, 2011) ${ }^{10}$. El juez Sajó, en su voto disidente, pone en duda el significado de la noción de «grupo vulnerable» como noción homogénea y pone de relieve las diferencias intragrupales, allanando el camino hacia una reconceptualización de la vulnerabilidad. En este sentido parecen apuntar las sentencias recientes del TEDH que consideran la «vulnerabilidad específica» de los demandantes.

10 Sobre la vulnerabilidad de los demandantes asilo, véase Morgades Gil (2010). 


\section{EL ENFOQUE DE LA INTERSECCIONALIDAD Y LA «VULNERABILIDAD ESPECÍFICA»}

Para evitar el riesgo de la homogeneización, debería evitarse considerar la vulnerabilidad como una condición permanente e inmutable y abordar su dimensión dinámica y estratificada (Luna, 2009). De acuerdo con Florencia Luna (2009), ninguna estructura social aislada es capaz de explicar la situación de vulnerabilidad que sufren las personas cuyos derechos han sido violados. Es una multiplicidad de «estratos» lo que hace vulnerable a una persona. Y si es verdad que considerar la «estratificación» añade un nivel de complejidad que es difícil de gestionar, este enfoque promete ser más útil y constructivo que fijar como vulnerables a grupos enteros (Luna, 2009: 331).

Desde finales de los setenta, las categorías sociales utilizadas de forma estanca y estática han sido criticadas porque no permitían dar cuenta de las interacciones de género, clase, raza, religión, sexualidad, edad y (dis)capacidad que definen nuestro posicionamiento social y (re)producen las (des)igualdades de forma entrelazada (Combahee River Collective, 1977; Hooks, 1981; Lorde, 1982; Davis, 1981; Spelman, 1988; Crenshaw, 1989; Hill Collins, 1990).

Una aproximación dinámica y estratificada a la vulnerabilidad permite dar cuenta de la vulnerabilidad de personas y grupos en un determinado contexto o momento histórico, sin asumir su vulnerabilidad como permanente. Además, permite reconocer que dentro de un grupo vulnerable, algunas personas pueden sufrir vulneración de derechos y otras no. Un concepto de vulnerabilidad dinámico y estratificado puede servir como herramienta para explorar de qué manera las estructuras sociales e institucionales (re)producen, y en ocasiones refuerzan, las situaciones de vulnerabilidad, a veces precisamente a través de aquellos instrumentos adoptados para prevenirla y eliminarla (Peroni y Timmer, 2013).

Este debate, introducido por académicas y activistas afroamericanas, se revitalizó a partir de 1989, cuando la jurista Kimberlé Crenshaw acuñó el término intersectionality para referirse a la vulnerabilidad que sufren las mujeres afroamericanas por encontrarse en el lado subordinado tanto de las estructuras de género como de las estructuras raciales en los Estados Unidos (Crenshaw, 1991: 1252). Su especial vulnerabilidad radica en el hecho de estar en la intersección entre dos sistemas de subordinación social y no puede explicarse sumando los efectos de un tipo de discriminación a otro. Se requiere prestar atención a los efectos de co-constitución (Lugones, 2012) de la subordinación específica de las mujeres afroamericanas en los Estados Unidos. Apuntar a las intersecciones sirve para contrarrestar «las tendencias de ver la raza y el género como exclusivos o separables» (Crenshaw, 1991: 1244-45) y permite prestar 
atención a las «diferencias que hacen la diferencia» a la hora de experimentar las vulneraciones de derechos (Crenshaw, 2000).

El concepto de interseccionalidad ha sido usado desde entonces para reivindicar la complejidad de los procesos que generan las desigualdades sociales. Centrándose en el posicionamiento social individual como un todo indisoluble (Anthias, 2002; Brah y Phoenix, 2004; Yuval-Davis, 2006; Nash, 2008; La Barbera, 2012), esta perspectiva analítica revela que las desigualdades son producidas por la interacción de estructuras sociales que se constituyen entre sí de forma dinámica y variada en el tiempo y en el espacio (La Barbera, 2017). La interseccionalidad permite entender el posicionamiento social como un lugar de subjetivación, poder y privilegio que cambia dinámicamente a lo largo del tiempo y en los distintos contextos sociopolíticos en relación con las diferentes estructuras sociales y representaciones discursivas que conforman las relaciones sociales (Winker y Degele, 2011; La Barbera, 2016). Centrándose en estas interacciones, la interseccionalidad revela la complejidad de la constitución mutua y simultánea tanto de la vulnerabilidad como de los privilegios. $\mathrm{Al}$ tener en cuenta que la vulnerabilidad está constituida por la interacción de distintas estructuras sociales, la interseccionalidad se aleja de los modelos explicativos de la vulnerabilidad meramente «aditivos» (Spelman, 1988; Hancock, 2007) y, apuntando a su dimensión estructural y sustantiva, trata de dar una respuesta integral a la complejidad de las desigualdades sociales de manera sustantiva (Bartlett et. al., 2002; Chamallas, 2003; Hannett, 2003; Fineman, 2008; Radacic, 2008; Young, 2009; Hunter, 2013, Barrère y Morondo, 2011; Bonthuys, 2013).

«Hacer la otra pregunta» (Matsuda, 1991) es crucial para desentrañar esta complejidad. Cuando se trata de una vulnerabilidad aparentemente vinculada a discriminaciones raciales, el enfoque de la interseccionalidad invita a preguntar de qué manera el sexismo es también relevante; si un problema se representa como relacionado con la discriminación de género, la interseccionalidad nos recuerda que debemos preguntar también sobre el heterosexismo; y así, cuando la vulnerabilidad parece estar vinculada a la homofobia, la interseccionalidad llama a indagar sobre su conexión con el clasismo (La Barbera, 2017).

La interseccionalidad está siendo progresivamente incorporada en el derecho internacional de los derechos humanos. El Comité para la Eliminación de la Discriminación contra la Mujer afirma que

la discriminación de la mujer por motivos de sexo y género está unida de manera indivisible a otros factores que afectan a la mujer, como la raza, el origen étnico, la religión o las creencias, la salud, el estatus, la edad, la clase, la casta, la orientación sexual y la identidad de género. La discriminación por motivos de sexo o género 
puede afectar a las mujeres de algunos grupos en diferente medida o forma que a los hombres. Los Estados partes deben reconocer y prohibir en sus instrumentos jurídicos estas formas entrecruzadas de discriminación y su impacto negativo combinado en las mujeres afectadas (Recomendación General núm. 28 relativa a las obligaciones básicas de los Estados partes de conformidad con el artículo 2 de la Convención sobre la eliminación de todas las formas de discriminación contra la mujer, CEDAW/C/2010/47/GC.2, párr. 18).

Algunos tribunales han reconocido que «cuando existen dos causas de discriminación, éstas no pueden ser reducidas claramente a componentes distintos»: el intento de dividir la identidad de la parte demandante puede distorsionar o llevar a ignorar la naturaleza particular de su experiencia (Lam v University of Hawaii, USA, 1994). Asimismo, se ha argumentado que «la naturaleza de la discriminación es tal que no siempre puede ser compartimentada en categorías discretas de manera sensata. La discriminación es a menudo una experiencia multifacética» (Ministry of Defense v Tilern De Bique, UK, 2010). De esta manera, adoptar la interseccionalidad como criterio interpretativo en la praxis judicial ha posibilitado la protección de derechos que no estarían protegidos de otra forma.

Adoptando la interseccionalidad como criterio interpretativo propuesta por la demandante ${ }^{11}$ y las terceras partes intervinientes ${ }^{12}$, en B.S. c. España el $\mathrm{TEDH}$ ha reconocido que las distintas causas de discriminación interactúan y generan situaciones específicas de sobreexposición a la vulneración de derechos. En este caso, el Tribunal se refirió a la «vulnerabilidad específica» de la demandante - una mujer de origen nigeriano que ofrecía servicios sexuales en la calle - como el resultado de la intersección de su raza, género y situación sociolaboral. Haciendo referencia a la «vulnerabilidad específica» de la demandante, sin vincularla a un presunto grupo de pertenencia, el TEDH ha evitado la esencialización de la situación de vulnerabilidad y el consiguiente estigma que caracteriza gran parte de la jurisprudencia previa del Tribunal en materia de personas y grupos vulnerables. La categoría de la «vulnerabilidad específica» permite al TEDH reconocer que las personas se vuelven particularmente vulnerables debido a un conjunto de causas dinámicamente relacionadas

11 La demandante, Beauty Solomon, fue representada legalmente por Women's Link Worldwide, una asociación sin ánimo de lucro con sede en Madrid especializada en violaciones de los derechos de las mujeres desde una perspectiva interseccional. Véase http://www.womenslinkworldwide.org/.

12 El Advice on Individual Rights in Europe Centre (AIRE), en colaboración con la Sexuality and Gender Law Clinic de la Columbia Law School y la European Social Research Unit (ESRU) de la Universidad de Barcelona. 
entre ellas, incluyendo las desventajas económicas, los prejuicios sociales y las características físicas, concretas y contextuales (La Barbera, 2016). La adopción de la interseccionalidad como criterio interpretativo de la situaciones de vulnerabilidad tiene profundas implicaciones en la praxis jurídica: en primer lugar, permite centrarse en la interacción entre las distintas a causas que determinan la vulnerabilidad; en segundo lugar, facilita la consideración de los efectos perversos de la legislación y de la praxis en términos de reproducción de privilegios y estigma (La Barbera, 2017); finalmente, ayuda a apuntar a remedios específicos que no son previsibles de manera abstracta y apriorística. Sin embargo, en B.S. c. España el TEDH limita los efectos transformativos de su decisión al no indicar las medidas concretas que España hubiese tenido que adoptar para evitar en el futuro violaciones de derechos análogas.

\section{CONCLUSIONES}

El discurso de la vulnerabilidad tiene cada vez más relevancia para el TEDH. Sin embargo, el Tribunal no utiliza la categoría de la vulnerabilidad de forma consistente, refiriéndose a "personas vulnerables», "grupos vulnerables», «los más vulnerables», «especialmente vulnerables», «marginados», «desfavorecidos» $\mathrm{y}$ «menos privilegiados» sin distinción aparente. A pesar de su creciente difusión, la categoría de la vulnerabilidad se encuentra todavía en construcción. El discurso de la vulnerabilidad en la jurisprudencia del TEDH muestra un vínculo con el daño o riesgo de sufrirlo. La pertenencia a un grupo vulnerable es para el Tribunal una característica explicativa de las violaciones de derechos humanos sufridas por determinados colectivos desaventajados. Desde luego, para el TEDH el concepto de grupo asume una relevancia especial. El Tribunal asume que existen características compartidas que diferencian entre quienes se representan como integrantes de un grupo vulnerable de la población en general, ubicando a los primeros en una situación de mayor desventaja.

La potencialidad que entraña el uso de la categoría de la vulnerabilidad en la praxis judicial es la capacidad de apuntar a las causas estructurales que determinan las violaciones sistemáticas de derechos, abriendo así el camino hacia un abordaje de las violaciones de derechos en su dimensión sustantiva (Al Tamimi, 2016) y transformativa (Fredman, 2016). Sin embargo, la vulnerabilidad se queda vacía como categoría en todas aquellas - no pocasocasiones en las que el TEDH no menciona en el fallo las causas estructurales identificadas en los antecedentes. El Tribunal no indica en la mayoría de los casos las medidas que los Estados tienen que adoptar para evitar las violaciones de derechos y combatir sus causas sistémicas. El Tribunal ha afirmado solo en contadas ocasiones que cuando personas que pertenecen a un grupo vulnerable 
sufren una violación de derechos es necesario otorgarles una protección especial y reducir el margen de apreciación del Estado condenado. Esta limitación procedural se acompaña de otra de naturaleza más conceptual relacionada con la falta de definición de la relación entre causas estructurales, noción de grupo social y violación de derecho individual. La noción de grupo vulnerable utilizada por el TEDH, en primer lugar, oculta que las características que definen la posición social no son inmutables y fijas; en segundo lugar, ignora la existencia de diferencias significativas entre quienes se representan como sus integrantes así como la dimensión entrelazada de la posición social de quien sufre una violación de derechos; y finalmente, produce el efecto paradójico de conducir a aproximaciones victimizadoras y estigmatizadoras que terminan por incrementar la vulnerabilidad que aspira a eliminar o compensar.

Para evitar estos efectos perversos, es crucial que la vulnerabilidad no se considere como categoría estática, sino como el resultado de la interacción de estructuras sociales, políticas y discursivas. En este sentido, el uso de la interseccionalidad como criterio interpretativo puede ayudar a corregir los efectos perversos del uso de la categoría de la vulnerabilidad en la praxis judicial del TEDH. La adopción de la categoría de la «vulnerabilidad específica» a partir de un enfoque interseccional ha permitido al Tribunal reconocer que las personas se vuelven particularmente vulnerables debido a un conjunto dinámico de causas que interactúan entre ellas generando situaciones específicas de sobreexposición a la violación de los derechos que necesitan protección especial por parte del Estado.

Utilizar la interseccionalidad como criterio interpretativo de las situaciones de vulnerabilidad de las partes demandantes permite perseguir una doble finalidad. Por un lado, identificar las relaciones de poder y privilegio que conforman las estructuras colectivas donde se articula la exposición subjetiva al riesgo de sufrir vulneraciones de derechos; por el otro, revelar las interacciones dinámicas entre las distintas estructuras que definen el posicionamiento individual dentro de los grupos sociales identificados como vulnerables. De este modo, el enfoque de la interseccionalidad se presenta como una herramienta de suma utilidad para superar los límites conceptuales de la categoría de la vulnerabilidad y explotar su potencialidad para proteger y garantizar los derechos humanos en los países miembros del Consejo de Europa.

\section{Bibliografía}

Al Tamimi, Y. (2016). The Protection of Vulnerable Groups and Individuals by the European Court of Human Rights. Journal européen des droits de l'homme/European Journal of Human Rights, 5, 561-583. 
Anderson, M. (1994). El concepto de vulnerabilidad: más allá de la focalización en los grupos vulnerables. Revista Internacional de la Cruz Roja, 19, 336-341.

Anthias, F. (2002). Beyond feminism and multiculturalism: Locating difference and the politics of location. Women's Studies International Forum, 25(3), 275-286. Disponible en: https://doi.org/10.1016/S0277-5395(02)00259-5.

Bacchi, C. (1999). Women, Policy and Politics: the construction of policy problems. London: Sage.

Barranco Avilés, M. C. y Churruca Muguruza, C. (eds.). (2014). Vulnerabilidad y protección de los derechos humanos. Valencia: Tirant lo Blanch.

Barranco Avilés, M. C. (2014). Derechos Humanos y vulnerabilidad. Los ejemplo del sexismo y el edadismo. En M. C. Barranco Avilés y C. Churruca Muguruza (eds.). Vulnerabilidad y protección de los derechos humanos (pp. 17-44). Valencia: Tirant lo Blanch.

- (2016). Vulnerabilidad, derechos humanos y empresas, Cuadernos Electrónicos de Filosofía del Derecho, 34, 1-16. Disponible en: https://doi.org/10.7203/ CEFD.34.8925.

Barrère Unzueta, M. y Morondo Taramundi, D. (2011). Subordiscriminación y discriminación interseccional: elementos para una teoría del derecho antidiscriminatorio. Anales de la Cátedra Francisco Suárez, 45, 15-42.

Barrère Unzueta, M. (2016). Martha A. Fineman y la igualdad jurídica: ¿Vulnerabilidad vs. Subordiscriminación? Cuadernos Electrónicos de Filosofía del Derecho, 34, 17-34. Disponible en: https://doi.org/10.7203/CEFD.34.8927.

Bartlett, K, Harris, H. y Rhode, D. (2002). Gender and Law: Theory, Doctrine, Commentary. New York: Aspen.

Bonthuys, E. (2013). Equality and Difference: Fertile Tensions or Fatal Contradictions for Advancing the Interests of Disadvantaged Women? En V. Munro y M. Davies (eds.). The Ashgate Research Companion to Feminist Legal Theory (pp. 85-104). Farnham: Ashgate.

Brah, A. y Phoenix, A. (2004). Ain't I a woman? Revisiting intersectionality. Journal of International Women's Studies, 5(3), 75-86.

Butler, J. (1990). Gender Trouble: Feminism and the Subversion of Identity. New York: Routledge.

- (2004). Precarious life: The powers of mourning and violence. London: Verso.

Chamallas, M. (2003). Introduction to Feminist Legal Theory. New York: Aspen.

Chapman, A. y Carbonetti, B. (2011). Human Rights Protections for Vulnerable and Disadvantaged Groups: The Contributions of the UN Committee on Economic, Social and Cultural Rights. Human Rights Quarterly, 33(3), 682-732.

Clayton, G. (2011). Asylum Seekers in Europe: M. S. S. v Belgium and Greece. Human Rights Law Review, 11 (4), 758-773, 3. Disponible en: https://doi. org/10.1093/hrlr/ngr037.

Combahee River Collective [1977] (1986). Combahee river collective statement. Black feminist organizing in the seventies and eighties. Albany: Women of Color Press. 
Crenshaw, K. (1989). Demarginalizing the Intersection of Race and Sex: A Black Feminist Critique of Antidiscrimination Doctrine, Feminist Theory and Antiracist Politics. University of Chicago Legal Forum, 139-167.

- (1991). Mapping the margins: Intersectionality, identity politics, and violence against women of color. Stanford Law Review, 43, 1241-1299.

(2000). Gender-related aspects of race discrimination (EGM/GRD/2000/WP.1). Background paper for the United Nations Expert Meeting on "Gender and Racial Discrimination", November 21-24, Zagreb, Croatia.

Da Lomba, S. (2014). Vulnerability, Irregular Migrants' Health-Related Rights and the European Court of Human Rights. European Journal of Health Law, 21, 339-364.

Davis, A. (1981). Women, Race and Class. New York: Vintage.

Estupiñan Silva, R. (2014). La vulnerabilidad en la jurisprudencia de la Corte Interamericana de Derechos Humanos: esbozo de una tipología. En L. Burgorgue-Larsen, A. Maués y B. Eugenia Sánchez Mojica (eds.). Derechos Humanos y Politicas Públicas (pp. 193-231). Barcelona: EDO.

Fineman, M. (2008). The vulnerable subject: Anchoring equality in the human condition. Yale Journal of Law and Feminism, 20, 1-23.

- (2010). The Vulnerable Subject and the Responsive State. Emory Law Journal, $60,251-275$.

(2013). Equality, Autonomy, and the Vulnerable Subject in Law and Politics. En M. Fineman y A. Grear (eds.), Vulnerability: Reflections on a New Ethical Foundation for Law and Politics (pp. 13-26). Farnham: Ashgate.

Fitzgerald, S. (2010). Biopolitics and the regulation of vulnerability: the case of the female trafficked migrant. International Journal of Law in Context, 6(3), 277294. Disponible en: https://doi.org/10.1017/S1744552310000169.

Fredman, S. (2016). Substantive Equality Revisited. International Journal of Constitutional Law, 14 3), 712-738.

Gilligan, C. (1982). In a Different Voice: Psychological Theory and Women's Development. Cambridge (MA): Harvard University Press.

Grear, A. (2013). Framing the project of international human rights law: reflections on the dysfunctional family of the Universal Declaration. En C. Gearty y C. Douzinas (eds.). The Cambridge Companion to Human Rights Law (pp. 17-35). Cambridge: Cambridge University Press.

Gyorfi, T. (en prensa). The legitimacy of the European human rights regime. A view from the United Kingdom. Global constitutionalism.

Hancock, A. (2007). When multiplication doesn't equal quick addition: Examining intersectionality as a research paradigm. Perspectives on Politics, 5(1), 63-79. Disponible en: https://doi.org/10.1017/S1537592707070065.

Hannett, S. (2003). Equality at the intersections: The legislative and judicial failure to tackle multiple discrimination. Oxford Journal of Legal Studies, 23(1), 65-86. Disponible en: https://doi.org/10.1093/ojls/23.1.65.

Hill Collins, P. (1990). Black Feminist Thought. Knowledge, consciousness, and the politics of empowerment. London: Routledge. 
Hooks, B. (1981). Ain't I a Woman: Black Women and Feminism. Boston: South End Press.

Hunter, R. (2013). Contesting the Dominant Paradigm: Feminist Critiques of Liberal Legalism. En V. Munro y M. Davies (eds.). The Ashgate Research Companion to Feminist Legal Theory (pp. 13-30). Farnham: Ashgate.

Keogh, M., Fox, N. y Flynn, E. (2010). How Far Towards Equality? A Vulnerabilities Approach to the Rights of Disabled People. Working Papers in Law, Criminology \& Socio-Legal Studies, 29. Disponible en: https://doi.org/10.1080/09687599. 2015.1113162.

La Barbera, MC. (2012). Intersectional-gender and the Locationality of Women in Transit. En G. Bonifacio (ed.), Feminism and Migration: Cross-Cultural Engagements (pp. 17-31). Dordrecht: Springer.

La Barbera, MC. (2016). Interseccionalidad, un «concepto viajero»: orígenes, desarrollo e implementación. Revista InterDisciplina 4 (8), 103 - 120.

La Barbera, MC. (2017). Intersectionality and its journeys: from counterhegemonic feminist theories to law of European multilevel democracy. Investigaciones Feministas, 8(1), 133-149. Disponible en: http://dx.doi.org/10.5209/INFE.54858.

Lorde, A. (1982). Zami: A new spelling of my name. Watertown: Persephone Press.

Lugones, M. (2012). Subjetividad esclava, colonialidad de género, marginalidad y opresiones múltiples. En VV. AA. Pensando los feminismos en Bolivia (pp. 129139). La Paz: Conexión Fondos de Emancipación.

Luna, F. (2009). Elucidating the Concept of Vulnerability: Layers not Labels. International Journal of Feminist Approaches to Bioethics, 2 (1), 121-139. Disponible en: https://doi.org/10.3138/ijfab.2.1.121.

Matsuda, M. (1991). Beside my sister, facing the enemy: Legal theory out of coalition. Stanford Law Review, 43, 1183-1192.

Morgades Gil, S. (2010). La protección de los demandantes de asilo por razón de su vulnerabilidad especial en la jurisprudencia del tribunal Europeo de los derechos humanos. Revista de Derecho Comunitario Europeo, 37, 801-842.

Morondo Taramundi, D. (2016). ¿Un nuevo paradigma para la igualdad? La vulnerabilidad entre condición humana y situación de indefensión. Cuadernos Electrónicos de Filosofía del Derecho, 34, 205-221. Disponible en: https://doi. org/10.7203/CEFD.34.8916.

Munro, V. y Scoular, J. (2012). Abusing Vulnerability? Contemporary Law and Policy Responses to Sex Work in the UK. Feminist Legal Studies, 20, 189-206. Disponible en: http://dx.doi.org/10.1007/s10691-012-9213-x.

Nash, J. (2008). Re-thinking Intersectionality. Feminist Review, 89, 1-15.

Nussbaum, M. (1999). Sex and Social Justice. Oxford: Oxford University Press.

Okin, S. (1999). Is Multiculturalism Bad for Women? En J. Cohen, M. Howard y M. Nussbaum (eds.), Is Multiculturalism Bad for Women? (pp.7-24). Princeton: Princeton University Press.

Omi, M. y Winant, H. (1986). Racial Formation in the United States: From the 1960s to the 1980s. New York: Routledge. 
Pateman, C. (1983). Feminist critiques of the public/private dichotomy. En S. Benn y G. Gaus (eds.). Public and Private in Social Life (pp. 281-303). London: Croom Helm.

Peroni, L. y Timmer, A. (2013). Vulnerable groups: The promise of an emerging concept in European Human Rights Convention law. International Journal of Constitutional Law, 11(4), 1056-1085. Disponible en: https://doi.org/10.1093/ icon/mot042.

Perry, I. (2011). More Beautiful and More Terrible: The Embrace and Transcendence of Racial in the United States. New York: New York University Press.

Phillips, A. (2010). What's wrong with Essentialism? Distinktion: Scandinavian Journal of Social Theory, 11(1), 47-60. Disponible en: https://doi.org/10.1080/160 0910X.2010.9672755.

Radacic, I. (2008). Gender equality Jurisprudence of the European Court of Human Rights. The European Journal of International Law, 19(4), 841-857. Disponible en: https://doi.org/10.1093/ejil/chn044.

Rodríguez Díaz, S. y Ferreira, M. (2010). Desde la dis-capacidad hacia la diversidad funcional. Un ejercicio de dis-normalización. Revista Internacional de Sociología, 68, 289-309. Disponible en: https://doi.org/10.3989/ris.2008.05.22.

Scott, J. W. (1988). Deconstructing equality-versus-difference: Or, the uses of poststructuralist theory for feminism. Feminist Studies, 14(1), 32-50. Disponible en: http://dx.doi.org/10.2307/3177997.

Solanke, I. (2016). Discrimination as Stigma: A Theory of Anti-discrimination Law. Oxford: Bloomsbury Publishing.

Spelman, E. (1988). Inessential Woman: Problems of Exclusion in Feminist Thought. Boston: Beacon.

Timmer, A. (2013). A Quiet Revolution: Vulnerability in the European Court of Human Rights. En M. Fineman y A. Grear (eds.). Vulnerability: Reflections on a New Ethical Foundation for Law and Politics (pp. 147-170). Farnham: Ashgate.

Tronto, J. (1993). Moral Boundaries: A Political Argument for an Ethic of Care. New York: Routledge.

Truscan, I. (2013). Considerations of vulnerability: from principles to action in the case law of the European Court of Human Rights, Retfard: Nordic Journal of Law and Justice, 142 (3), 64-83.

Viola, F. (2000). Etica e metaetica dei diritti umani. Torino: Giappichelli.

Winker, G. y Degele, N. (2011). Intersectionality as multi-level analysis: Dealing with social inequality. European Journal of Women's Studies, 18, 51-66.

Young, I. M. (1990). Justice and the politics of difference. Princeton: Princeton University Press.

- (2009). Structural injustice and the politics of difference. En E. Grabham, D. Cooper, J. Krishnadas, D. Herman (eds.). Beyond intersectionality: Law, power and the politics of location (pp. 273-299). New York: Routledge and Cavendish.

Yuval-Davis, N. (2006). Intersectionality and feminist politics. European Journal of Women's Studies, 13(3), 193-209.Disponibleen:https://doi.org/10.1177/1350506806065752. 